\title{
A SYNCHRONIZED FEL-SYNCHROTRON RADIATION FACILITY AT JEFFERSON LAB
}

\author{
A. Hutton", S. V. Benson, H. F. Dylla, O. Garza, R. R. Lauzé, R. T. May, G. R. Neil, S. L. Prior, \\ G. P. Williams, N. W. Wilson, Jefferson Lab, 12000 Jefferson Avenue, Newport News, VA23606
}

\section{Abstract}

Jefferson Lab is planning a facility for studying ultrafast dynamical processes using a $1 \mathrm{~kW}$ average power IR/UV FEL, and a mode-locked Ti-sapphire femtosecond laser in the visible, combined with a $1 \mathrm{~nm}$ critical wavelength electron storage ring. Light pulses from the three sources will be synchronized at $125 \mathrm{MHz}$ for pumpprobe studies (including pump-pump-probe and pumpprobe-probe options) in chemistry, physics, materials science, medicine and biology. The FEL operates with pulses as short as 300 femtoseconds $(\sigma)$, which will provide the narrow bandwidth pump at high peak as well as average power. The FEL is currently operating and will soon be upgraded to operate at up to 10 kilowatt average power at an extended wavelength range from 300 $\mathrm{nm}$ to $10,000 \mathrm{~nm}$. A compact superconducting storage ring, Helios- 1 , has recently been donated to Jefferson Lab that has a critical wavelength of $10 \AA$ and is capable of storing currents up to $800 \mathrm{~mA}$. In addition to providing spectroscopy capabilities, the storage ring will also support exploratory $\mathrm{x}$-ray lithography $\mathrm{R} \& \mathrm{D}$, including a precision stepper-aligner for training purposes. The facility, which is expected to become available in 2004 , will be described and the capabilities detailed.

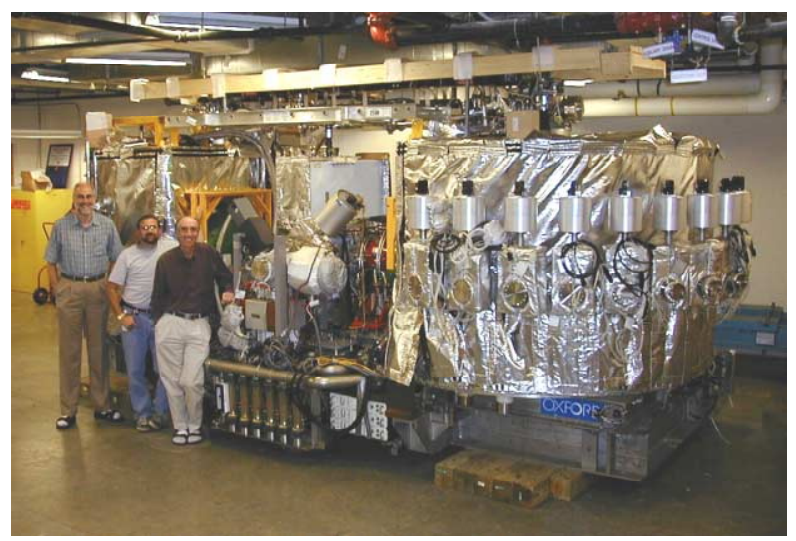

Fig. 1. The superconducting electron storage ring Helios1 at Jefferson Lab. The synchrotron radiation ports are sealed by valves and can be seen on the right of the photo. L - R. Andrew Hutton, Ron Lauzé and Gwyn Williams.

\footnotetext{
* This work was supported by US DOE Contract No. DE-AC05-84ER40150, the Office of Naval Research, the Commonwealth of Virginia, and the Laser Processing Consortium.
}

\section{OVERVIEW}

Jefferson Lab operates a world "best in class" freeelectron laser (FEL)[1] which is based on a photo-injected dc gun, and a superconducting radio frequency, energyrecovered linac (SRF linac). The laser is based on a nearconcentric optical resonator cavity surrounding a wiggler and pumped by radiation emitted by a quasi-continuous electron beam from the SRF linac. Accelerator operations provide routine, reliable, delivery of light to one of six labs. The laser operates in the range from 3-7 microns in the fundamental, with sub picosecond pulses containing up to $20 \mu$ Joules of energy each and at a repetition rate of $18.7,37.4$ or $74.85 \mathrm{MHz}$ delivering a maximum average power up to $1.0 \mathrm{~kW}$ to users. The high beam power in a very tightly focused spot with a variable wavelength makes the FEL an ideal pump for pump-probe experiments.

In addition, one can consider a mode locked CPA Tisapphire short pulse laser in the terawatt peak power range @ $10 \mathrm{~Hz}$ repetition rate as another pump source in the visible for processes with energetics complementary to infrared pumps. Alternatively, one can consider using a $10 \mathrm{kHz}-1 \mathrm{MHz}$ Ti-sapphire laser with energies per pulse in the tens of microjoules to be used either as a probe in the visible or as an "energy modulator" and "slicer" [2] to reach down further into the ultrafast domain from femtoto atto-second regime. This would allow studies of exotic atomic physics such as coherent ionization and collapse/entanglement of quantum wave functions.

The Helios-1 compact synchrotron operates at 700 $\mathrm{MeV}$ with stored beam currents up to $800 \mathrm{~mA}$. With 4.5 Tesla superconducting bending magnets, Helios-1 produces more than $30 \mathrm{~kW}$ of synchrotron radiation in the $\mathrm{x}$-ray to IR range. Since the spectrum is flat over a broad wavelength range, Helios- 1 is a perfect probe for pumpprobe experiments.

\section{PUMP-PROBE EXPERIMENTS}

Pump-probe measurements using a synchrotron radiation source synchronized to a mode-locked Tisapphire laser have been demonstrated at the NSLS, Brookhaven [3,4] and LBL [5]. The success of these and other experiments [6] motivated the present effort, which will offer higher peak power, and tunable light for the pump with shorter pulses for the probe $(\sigma=30$ ps compared to $300 \mathrm{ps}$ for the NSLS). Note that the technique applies to processes that repeat and recover, such as electron and vibrational relaxation processes, and chemical reactions in which the recovery is by replenishment. The use of a pulsed probe source 
establishes the time resolution, and the technique does not require a fast detector. Measurements are signal averaged with a fixed time delay between pump and probe and the experiment repeated for a range of such delays.

In addition, one can envision various other $\mathrm{P} 3$ processes (pump-pump-probe, pump-probe-probe) to study nonlinear echo and higher order decoherence and mixing effects in "complex" systems, where the complexity is not due to the complication of the very many subsystems, but rather due to the essential higher order coupling and correlations in an adaptive system such as the protein molecule. The versatility of such a capability lies in three color "IR"-"visible"-"X-ray" experiments in the P3 mode where, in addition, one or two of the pumps or probes could be either based on light beams or electron beams. The electron beam has the advantage of having a higher cross-section beneficial for studying fast chemical kinetics with weaker fluxes of probes.

\section{FEL CHARACTERISTICS}

The main measured characteristics of the FEL electron beam are given in Table 1.

Table 1: Measured FEL Electron Beam Parameters

\begin{tabular}{ll}
\hline Parameter & Measured value \\
\hline Electron beam energy & $48 \mathrm{MeV}$ \\
Average current & $4.8 \mathrm{~mA}$ \\
Bunch charge & Up to 60 pC \\
Bunch length (rms) & $0.4 \pm 0.1 \mathrm{ps}$ \\
Peak current & Up to 60A \\
Trans. emittance (rms) & $7.5 \pm 1.5 \mathrm{~mm} \mathrm{mr}$ \\
Long. emittance (rms) & $26 \pm 7 \mathrm{keV} \mathrm{deg}$ \\
Pulse repetition frequency & $18.7 \mathrm{MHz}$ (up to 74.8 MHz) \\
\hline \hline
\end{tabular}

The associated measured characteristics of the FEL light are shown in Table 2.

Table 2: Measured FEL Light Parameters

\begin{tabular}{|l|l|}
\hline Wavelength & $3-6.2 \mu \mathrm{m}$ in fundamental \\
\hline Average Power to users & Up to $1.0 \mathrm{~kW}$ \\
\hline Pulse Length & $0.3-1.7 \mathrm{ps}$ \\
\hline Pulse Repetition Freq. & $74.85,37.425,18.7 \mathrm{MHz}$ \\
\hline Micro pulse energy & Up to $25 \mu \mathrm{J}$ oules \\
\hline Bandwidth & $0.3-2 \%$ \\
\hline Polarization & $>6000: 1$ \\
\hline Transverse mode & $<2 \times$ diffraction limit \\
\hline Beam diameter in lab & $1.5-3.5 \mathrm{~cm}$ \\
\hline Amplitude jitter & $<10 \% \mathrm{p}-\mathrm{p}$ \\
\hline
\end{tabular}

The FEL Upgrades are funded in two separate packages. The first, which is well under way, is anticipated to be complete by October 2002. The FEL will be upgraded to operate in a wider wavelength range, namely from 0.30 microns in the ultraviolet, to 15 microns and with average powers up to $10 \mathrm{~kW}$. In the upgraded machine the energy will be increased from 40 $\mathrm{MeV}$ to $160 \mathrm{MeV}$ by the addition of two superconducting linac modules, the average beam current will be increased from 5 to $10 \mathrm{~mA}$ and the extraction efficiency will be increased by a factor of two using an optical klystron. A separate optical cavity and wiggler will be used for the ultraviolet region.

The second upgrade calls for the installation of the superconducting compact storage ring Helios-1, (see next section) to provide synchrotron radiation with light pulses synchronized with those from the FEL. To provide oneon-one synchronization, the optical cavity will have to be lengthened to provide for a pulse repetition rate of $\sim 125$ $\mathrm{MHz}$, which is the $12^{\text {th }}$ sub-harmonic of the $\sim 1500 \mathrm{MHz}$ linac frequency.

\section{HELIOS CHARACTERISTICS}

Helios is a superconducting electron storage ring, which has an electron beam energy of $700 \mathrm{MeV}$ and a critical wavelength of $10 \AA$. Built by Oxford Instruments, it is a racetrack machine with overall dimensions of $\sim 3 \times 5$ meters and a machine circumference of 9.6 meters. Beam is injected into the machine from a $100 \mathrm{MeV}$ linac, captured and then accelerated to the full energy of 700 $\mathrm{MeV}$. The energy is limited by the maximum magnetic field of the superconducting dipoles of 4.5 Tesla, which imposes a bending radius of 0.519 meters. Each $180^{\circ}$ dipole is equipped with ten ports for extracting the synchrotron light. With a radio frequency of $\sim 500 \mathrm{MHz}$ the machine operates at a harmonic number of 16. Filling only four symmetrically spaced bunches yields a pulse frequency of $125 \mathrm{MHz}$.

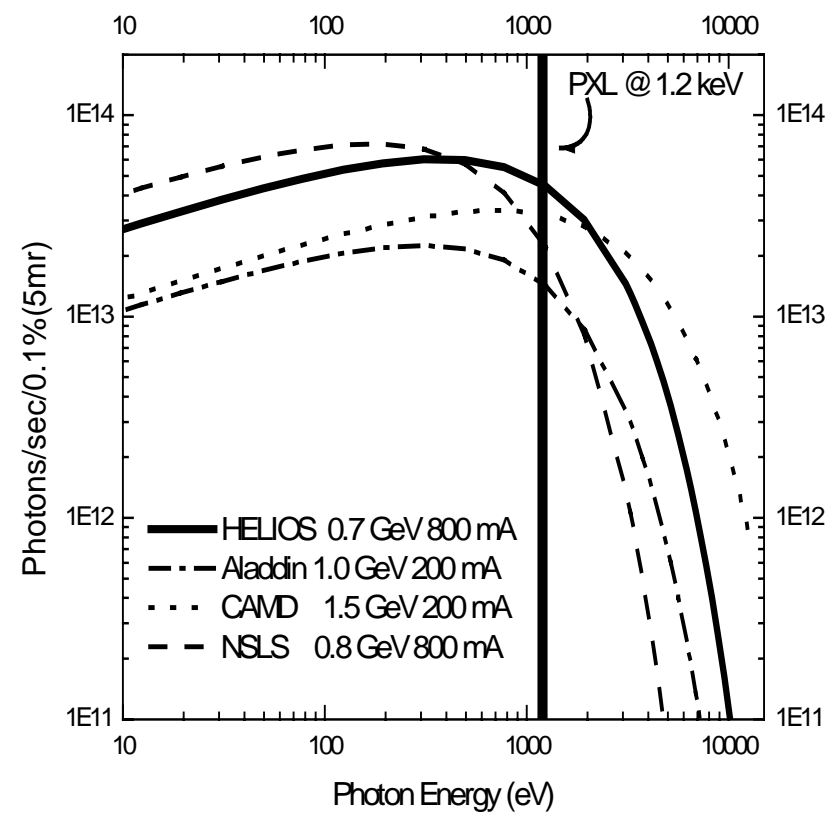

Fig. 2. Calculated output spectra of several electron storage rings that have been used for proximity $\mathrm{x}$-ray lithography. Aladdin is at the University of Wisconsin, Madison, CAMD is at Louisiana State University, Baton Rouge, and the NSLS is at Brookhaven National Laboratory, Upton, New York. 
Since the primary wavelength for PXL is $1 \mathrm{~nm}$, or 1.2 $\mathrm{keV}$, and since PXL does not require an ultra-low emittance source, Helios-1 is seen to have an ideal design.

Helios was designed for proximity X-ray lithography (PXL) and compares very favorably with other storage rings on which lithography is conducted as can be seen from the calculated output spectrum shown in Fig. 2.

In addition to its potential use for PXL, other uses of the storage ring as a stand-alone device are envisaged including IR, VUV and soft $x$-ray spectroscopy. The addition of a superconducting wiggler, for example, could provide harder $\mathrm{x}$-rays of use for structure determinations.

\section{FACILITY INTEGRATION}

In order to provide two-color, three-color, or pumpprobe capabilities based on synchronous operation of the JLab FEL, a short pulse laser, and Helios-1, the three machines need to be housed in a common building, so that they can feed light beams into a common laboratory. In our case, there is sufficient space in the existing FEL building to house the linac, the additional shielding that it requires, most of the power supplies and possibly a Tisapphire laser. The proposal is to add a 2-storey building addition of approximately $120 \times 120 \mathrm{ft}$. that would house Helios-1, the synchrotron radiation beamlines, and additional set-up space. The FEL and laser beam will be brought into proximity with the Helios- 1 beam, since it is easier to transport IR than X-ray beams.

We show a schematic of the combined facility in Fig. 3. On this drawing, we also show some infrared and soft-xray beamlines to set a scale.

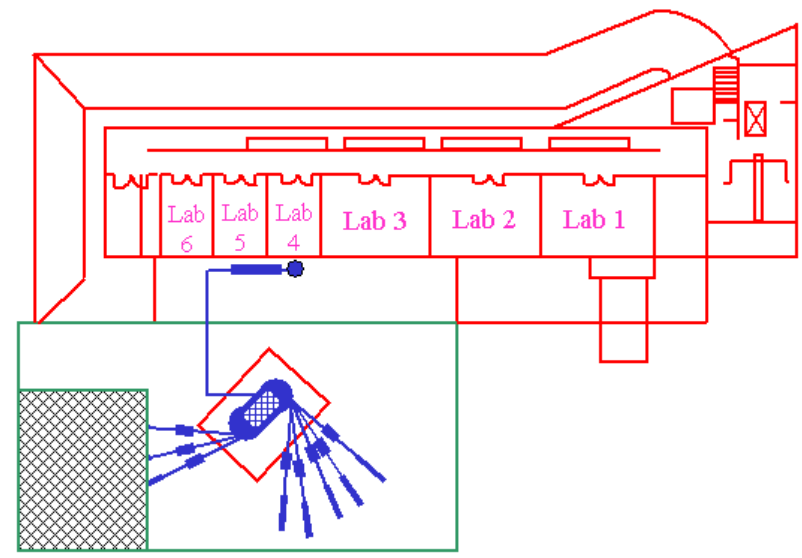

Fig. 3. Layout of Helios-1 in a building addition extending out from the Jefferson Lab Free Electron Laser building at the lower left. The hatched area within the addition is a clean area for $\mathrm{x}$-ray lithography. The labs at the top belong to the FEL and a beam from the lab area will be transported to the floor of the Helios-1 addition.

\section{CONCLUSION}

In conclusion, we are in the process of establishing a synchronized visible laser/FEL/synchrotron radiation facility. Examples of experiments that will be enabled by this facility are relaxation processes in the 100 ps to 100 attosecond time-scale such systems as semiconductors, superconductors, adsorbates at surfaces, and certain photochemical and photo-biological reactions.

In addition to the synchrotron radiation capabilities, we note that the FEL produces Thompson-scattered $\mathrm{x}$ rays in the 5-30 keV range with sub picosecond pulse lengths, and a peak brightness of $10^{10}$ photons $/ \mathrm{sec} / 0.1 \%$ bandwidth $/ \mathrm{mm}^{2} / \mathrm{mrad}^{2}$. Eventually this will increase to > $10^{11}$ photons $/ \mathrm{sec} / 0.1 \%$ bandwidth $/ \mathrm{mm}^{2} / \mathrm{mrad}^{2}$. These $\mathrm{x}$ rays are exactly synchronized with the IR pulses.

The short electron bunches also give rise to multi-particle coherent synchrotron radiation emission [5] in the far-IR or $\mathrm{THz}$ range. The average power produced in this range is $\sim 1$ watt $/ \mathrm{cm}^{-1}$, while the peak power is 30,000 times higher. This is to be compared with typical average powers of $10^{-6}$ watts $/ \mathrm{cm}^{-1}$ produced by conventional laser techniques. Again, these pulses are exactly synchronized with the IR pulses from the FEL and will extend the utility of this unique light source.

\section{ACKNOWLEDGEMENTS}

We would like to thank those that helped dismantle Helios-1 and move it to Jefferson Lab: R. Angello, D. Bruhewl, K. Cole, E. Diggs, D. Eardley, T. Fox, E. Hanson, J. Deihl, T. Menefee, E. Ratliff, S. Manning, S. Williams, K. Capek, M. Beck, R. Schwartz, J. Foyles, K. Mealer, I. Snowburg, J. Barbour, and K. Welch. We would also like to thank those that have been preparing the installation plans: S. Schwahn, S. Chandra, L. Even, C. Jones, C. Snetter, P. Powers, and A. Bogacz.

\section{REFERENCES}

1. G. R. Neil, C. L. Bohn, S. V. Benson, G. Biallas, D. Douglas, H. F. Dylla, R. Evans, J. Fugitt, A. Grippo, J. Gubeli, R. Hill, K. Jordan, G. Krafft, R. Li, , L. Merminga, P. Piot, J. Preble, M. Shinn, T. Siggins, R. Walker, and B. Yunn, Phys. Rev. Letts 84, 662 (2000).

2. R. Schoelein, S. Chattopadhyay, H.H. W.Chong, T.E. Glover, P. A. Heimann, C. V. Shank, A. A. Zholents and M. S. Zolotorev, Science, vol. 287, No. 5461, March 24, 2000, p. 2237.

3 G.L. Carr, R. P. S. M. Lobo, J. LaVeigne, D. H. Reitze, and D. B. Tanner, Phys. Rev. Letts 853001 (2000).

4. D.L. Ederer, J.E. Rubensson, D.R. Mueller, R. Shuker, W.L. O'Brien, J. Jai, Q.Y. Dong, T.A. Callcott, G.L. Carr, G.P. Williams, C.J. Hirschmugl, S. Etemad, A. Inam and D.B. Tanner, Nucl.Inst.\& Methods in Physics Research A319, 250 (1992).

5. R. Schoenlein et al., Science, 274, 11 Oct., 1996, p.236.

6. L. Nahon, E. Renault, M. Couprie, D. Nutarelli, D. Garzella, F. Polack, P. Dumas, G. Carr and G.P. Williams, SPIE Vol 3775145 (1999)

7. C. J. Hirschmugl, M. Sagurton and G. P. Williams, Physical Review A44, 1316, (1991). 